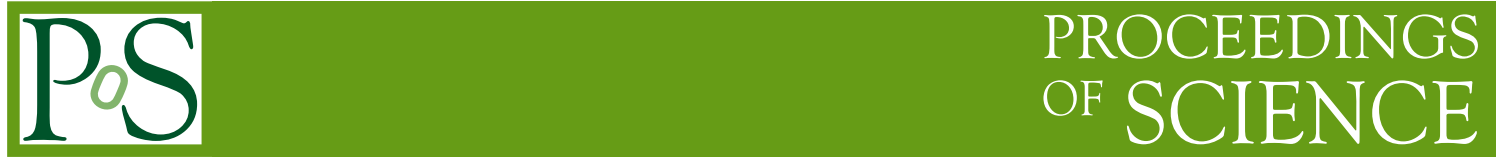

\title{
NC GUTS: A Status Report
}

\author{
C.P. Martin* \\ Departamento de Física Teórica I \\ Universidad Complutense de Madrid \\ Madrid-28040, Spain \\ E-mail: carmelodelbereth.fis.ucm.es
}

I review the main results that have been obtained so far on the construction of noncommutative GUTs

Corfu Summer Institute on Elementary Particles and Physics - Workshop on Non Commutative Field

Theory and Gravity

September 8 12, 2010

Corfu, Greece

\footnotetext{
${ }^{*}$ Speaker.
} 


\section{Introduction}

It is already ten years since the publishing of refs. [1-3], where it was put forward a formalism -called the enveloping-algebra formalism- which led to refs. [4, 5], where the Noncommutative Standard Model and noncommutative GUTs were formulated. An excellent introduction to noncommutative gauge theories defined within the enveloping-algebra formalism can be found in ref. [6].

Let us recall that in the enveloping-algebra formalism the noncommutative fields are functions of the ordinary fields -ie, no change in the number of degrees of freedom as we move from ordinary to noncommutative space-time- such that ordinary gauge orbits are mapped into noncommutative gauge orbits:

$$
\begin{aligned}
& A_{\mu}\left[a_{\mu}, \psi, \theta\right]+s_{N C} A_{\mu}\left[a_{\mu}, \psi, \theta\right] A=A_{\mu}\left[a_{\mu}+s a_{\mu}, \psi+s \psi, \theta\right], \\
& \Psi\left[a_{\mu}, \psi, \theta\right]+s_{N C} \Psi\left[a_{\mu}, \psi, \theta\right]=\Psi\left[a_{\mu}+s a_{\mu}, \psi+s \psi, \theta\right], \\
& s_{N C} \Lambda[\lambda, \lambda, \psi, \theta]=s \Lambda[\lambda, \lambda, \psi, \theta], \\
& A_{\mu}\left[a_{\mu}, \psi, \theta=0\right]=a_{\mu}, \Psi\left[a_{\mu}, \psi, \theta=0\right]=\psi, \Lambda[\lambda, \lambda, \psi, \theta=0]=\lambda \\
& s_{N C} A_{\mu}=\partial_{\mu} \Lambda-i\left[A_{\mu}, \Lambda\right]_{\star}, s_{N C} \Psi=i \Lambda \star \Psi, s_{N C} \Lambda=i \Lambda \star \Lambda, \\
& s a_{\mu}=\partial_{\mu} \lambda-i\left[a_{\mu}, \lambda\right], s \psi=i \lambda \psi, s \lambda=i \lambda \lambda,
\end{aligned}
$$

I shall call these equations standard Seiberg-Witten map equations since $\Lambda$ acts from the left on the matter fields $\Psi$. The solution to these equations which match the corresponding ordinary field when the noncommutativity matrix, $\theta^{\mu \nu}$, vanishes shall be called standard Seiberg-Witten map. Now, since $a_{\mu}$ and $\lambda$ take values on the Lie algebra, $\mathfrak{g}$, of a compact Lie group, $\mathrm{G}$, then, the noncommutative fields $A_{\mu}$ and $\Lambda$ take values on the universal enveloping algebra of $\mathfrak{g}$. This is a characteristic feature of noncommutative gauge fields defined in the enveloping-algebra formalism.

Having defined the noncommutative gauge and matter fields in terms of the ordinary fields using the solution to eq. (1.1), we now introduce de classical (nonSUSY) noncommutative GUT(inspired) theory for a compact Lie group, G, by giving its action $S$ :

$$
\begin{aligned}
& S=S_{\text {gauge }}+S_{\text {fermionic }}+S_{\text {Higgs }}+S_{\text {Yukawa }}, \\
& S_{\text {gauge }} \int d^{4} x-\frac{1}{2} \sum_{\mathscr{R}} c_{\mathscr{R}} \operatorname{Tr}_{\mathscr{R}} F_{\mu v}[\mathscr{R}(A)] \star F^{\mu v}[\mathscr{R}(A)], \\
& S_{\text {fermionic }}=\int d^{4} x \bar{\Psi}_{L} i \not D\left[\rho_{\psi}(A)\right] \Psi_{L}, \\
& S_{\text {Higgs }} \quad \text { and } \quad S_{\text {Yukawa }} \quad \text { give to, } \\
& F_{\mu v}[\mathscr{R}(A)]=\partial_{\mu} \mathscr{R}(A)_{v}-\partial_{\nu} \mathscr{R}(A)_{\mu}-i\left[\mathscr{R}(A)_{\mu}, \mathscr{R}(A)_{v}\right]_{\star}, \\
& D_{\mu}\left[\rho_{\psi}(A)\right] \psi_{L}=\partial_{\mu} \Psi_{L}-i \rho_{\psi}\left(A_{\mu}\right) \star \Psi_{L} .
\end{aligned}
$$

$S_{\text {Higgs }}$ and $S_{\text {Yukawa }}$ yield, respectively, the Higgs and Yukawa sectors of the GUT theory and are dropped to define what we call noncommutative GUT-inspired theories. We shall see later on that the construction of $S_{\text {Yukawa }}$ is far from trivial and it demands the use of the so-called Hybrid Seiberg-Witten maps [7] -needed to define noncommutative gauge transformation acting from the left and from right. In eq. (1.2), $\Psi_{L}\left[\theta^{\mu v}, \rho_{\psi}(a), \psi_{L}\right]$ is the noncommutative left-handed spinor multiplet which is the noncommutative counterpart of the ordinary left-handed spinor multiplet $\psi_{L} \cdot \psi_{L}$ carries an arbitrary unitary representation, $\rho_{\psi}$, of $\mathfrak{g} . \mathscr{R}$ labels the unitary irreps -typically the adjoint and matter irreps- of $\mathfrak{g}$ and $\sum_{\mathscr{R}} c_{\mathscr{R}} \operatorname{Tr}_{\mathscr{R}} \mathscr{R}\left(T_{\mathrm{I}}^{a}\right) \mathscr{R}\left(T_{\mathrm{I}}^{a}\right)=1 / g_{\mathrm{I}}^{2}$. 
Next, the quantum version of the classical field theory defined above is obtained by integrating over the ordinary fields in the path-integral with Boltzmann factor $e^{i S} . S$ is the action above, which we shall understand as a formal power series in $\theta^{\mu \nu}$. I believe that this expansion in $\theta$ will not yield the right Physics at Energies $>1 / \sqrt{\theta}$.

After those ten years, it is advisable that we pause to look back and assess what has been achieved as regards the quantum properties of those GUT(-inspired) theories. I will not cover all that has been done so far, but I will focus on

- Gauge anomalies.

- Renormalisability (when there are no Higgs and no Yukawa sectors),

- Construction of Yukawa terms.

- Existence of Supersymmetric versions.

\section{Gauge Anomalies}

When quantising a chiral gauge theory the first problem one has to face is that of gauge anomalies, for if the latter exist the theory will not make sense at the quantum level. The chiral vertices in the classical action acquire $\theta$-dependent terms, which can give rise to new $\theta$-dependent anomalous contributions to the famous ordinary triangle diagrams:

$$
S_{\text {fermionic }}=\int d^{4} x \bar{\psi} i \not \partial \psi+\bar{\psi}\left\{\not h-\theta^{\alpha \beta}\left[\frac{1}{2} f_{\alpha \beta} i \not D(a)+\gamma^{\rho} f_{\rho \alpha} i D_{\beta}(a)\right]\right\} \mathrm{P}_{L} \psi+o\left(\theta^{2}\right)
$$

Thus, I started long ago the computation of the three types of one-loop three-point diagrams in Figure 1 giving would-be $\theta$-dependent anomalies.
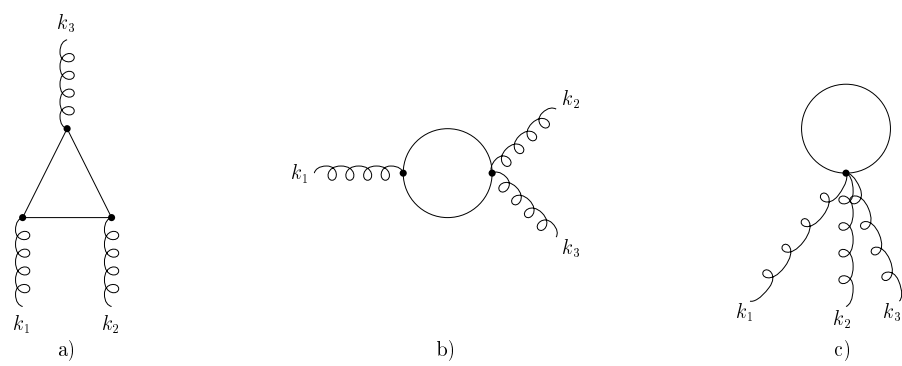

Figure 1: Types of $\theta$-dependent would-be anomalous three-point diagrams.

Actually, I was completely sure that these diagrams would give rise to new $\theta$-dependent anomalous terms, which would lead to extra anomaly cancelation conditions, which in turn would make most -eg., the Noncommutative Standard Model, noncommutative GUTs,...- of these theories meaningless at the quantum level. I could not be more mistaken! I was very surprised to find that the 
$\theta$-dependent anomalous contributions to the 1PI functional, $\Gamma$, were BRS-exact. ie, they were not truly anomalous terms:

$$
\begin{aligned}
& s \Gamma[A[a, \theta], \theta]=-\frac{i}{24 \pi^{2}} \int d^{4} x \varepsilon^{\mu_{1} \mu_{2} \mu_{3} \mu_{4}} \operatorname{Tr}\left(\partial_{\mu_{1}} \lambda a_{\mu_{2}} \partial_{\mu_{3}} a_{\mu_{4}}\right) \\
& +s\left[\frac{1}{48 \pi^{2}} \int d^{4} x \varepsilon^{\mu_{1} \mu_{2} \mu_{3} \mu_{4}} \theta^{\alpha \beta} \operatorname{Tr}\left(\partial_{\alpha} \partial_{\mu_{1}} a_{\mu_{2}} \partial_{\mu_{3}} a_{\mu_{4}} a_{\beta}\right)\right]+o\left(a^{3}\right)+o\left(\theta^{2}\right) .
\end{aligned}
$$

The computations that led to the previous results were carried out by using dimensional regularization with a nonanticommuting $\gamma_{5}$. More details can be found un ref. [8]. I would like to point out now that when I did the computations back in 2002, I was completely unaware of the results -obtained using cohomological techniques- by Barnich, Henneaux and Brandt on the lack on nonBardeen anomalies for semisimple Lie algebras. The result in eq. (2.1) holds, though, for nonsemisimple Lie algebras as well.

The next challenge was to show, at one-lop, that there were no $\theta$-dependent gauge anomalies at any order in $\theta$ and for any number of $a_{\mu}$ 's. We did so -see ref. [10], for details- by using a mixture of explicit Dimensional Regularization computations, brute force of BRS equations and cohomological BRS techniques. Indeed, by taking advantage of the fact that in Dimensional Regularization the Jacobian of $\mathscr{J}=\mathbb{I}+M$-an operator which enters the Seiberg-Witten map for fermions $\Psi_{\alpha I}=\left(\delta_{I J} \delta_{\alpha \beta}+M\left[a, \partial, \gamma, \gamma_{5} ; \theta\right]_{\alpha \beta I J}\right) \psi_{\beta J}$ is trivial, we were able to obtain the complete gauge anomaly candidate:

$$
\begin{aligned}
& \mathscr{A}[A, \Lambda, \theta]=-\frac{i}{24 \pi^{2}} \int d^{4} x \varepsilon^{\mu_{1} \mu_{2} \mu_{3} \mu_{4}} \operatorname{Tr} \Lambda \star \partial_{\mu_{1}}\left(A_{\mu_{2}} \star \partial_{\mu_{3}} A_{\mu_{4}}+\frac{1}{2} A_{\mu_{2}} \star A_{\mu_{3}} \star A_{\mu_{4}}\right), \\
& A_{\mu}=A[a, \theta]_{\mu}, \quad \Lambda=\Lambda[\lambda, \theta] .
\end{aligned}
$$

Then, by carrying out brute force computations and by using cohomological techniques, we obtained $\mathscr{B}\left[A^{(a, t \theta)}, t \theta\right]$ such that

$$
t \frac{d}{d t} \mathscr{A}[A(a, t \theta), \Lambda(\lambda, t \theta), t \theta]=s_{N C} \mathscr{B}\left[A^{(a, t \theta)}, t \theta\right]
$$

and, hence,

$$
\mathscr{A}[A(a, \theta), \Lambda(\lambda, \theta), \theta]=\mathscr{A}^{\text {Bardeen }}-s \int_{0}^{1} \frac{d t}{t} \mathscr{B}[A(a, t \theta), t \theta] .
$$

We thus concluded that the $\theta$-dependent contributions to $\mathscr{A}[A(a, \theta), \Lambda(\lambda, \theta), \theta]$ are cohomologically trivial: they are not anomalous contributions!

Since the previous result partially relies on the use a dimensionally regularised Feynman integrals involving $\gamma_{5}$, it would be advisable to check whether that result still holds for other regularization methods. Another way to obtain the gauge anomaly is Fujikawa's method: the gauge anomaly signals that the fermionic measure is not invariant under chiral gauge transformations. Fujikawa's method helps establish a connection with index theorems. As yet, we lack a derivation of the absence of $\theta$-dependent anomalous terms by using Fujikawa's method.

Within Fujikawa's formalism, the ordinary gauge anomaly comes in two guises, related by local redefinitions of the corresponding currents: the consistent form, $\mathscr{A}_{c o n}$, and the covariant form, $\mathscr{A}_{\text {cov }}$ :

- $\mathscr{A}_{c o n}$ verifies the Wess-Zumino consistency conditions and involves lengthy and tedious algebra. It is not gauge covariant. 
- $\mathscr{A}_{\text {cov }}$ does not verify the Wess-Zumino conditions, it is gauge covariant and, as a result, the algebraic computations that lead to it are simpler than in the "consistent" case.

As I was preparing a preliminary version of this talk, I decided to work out the covariant form, up to first order in $\theta$, of the gauge anomaly in the U(1) case. Let me point out that the absence of $\theta$-dependent contributions to the $U(1)$ gauge anomaly is nontrivial from the cohomological point of view of Barnich, Brandt and Henneaux. The results that I obtained are displayed next.

Let me begin with the following partition function

$$
\begin{aligned}
& Z[a, \theta] \equiv \int d \bar{\psi} d \psi \quad e^{-\int d^{4} x \bar{\psi} i \mathscr{D} \psi} \\
& \mathscr{D}=\hat{D}+\hat{R}, \quad \hat{D}=\not \partial-i \phi P_{L} \\
& \hat{R}=-\left[\frac{1}{4} \theta^{\alpha \beta} f_{\alpha \beta} \gamma^{\mu} D_{\mu}+\frac{1}{2} \theta^{\alpha \beta} \gamma^{\rho} f_{\rho \alpha} D_{\beta}\right] P_{L}
\end{aligned}
$$

Then, following Fujikawa, one introduces two bases of orthonormal eigenfunctions $\left\{\varphi_{m}\right\}$ and $\left\{\phi_{m}\right\}$

$$
(i \mathscr{D}(a))^{\dagger} i \mathscr{D}(a) \varphi_{m}=\lambda_{m}^{2} \varphi_{m}, \quad i \mathscr{D}(a)(i \mathscr{D}(a))^{\dagger} \phi_{m}=\lambda_{m}^{2} \phi_{m}
$$

and expands

$$
\psi=\sum_{m} a_{m} \varphi_{m}, \quad \bar{\psi}=\sum_{m} \bar{b}_{m} \phi_{\mu}^{\dagger}
$$

which leads to the following definition of the fermionic measure:

$$
d \bar{\psi} d \psi \equiv \prod_{m} d \bar{b}_{m} d a_{m}
$$

It is nor difficult to show that the gauge anomaly equation in covariant disguise reads

$$
\int d^{4} x \operatorname{Tr} \omega(x)\left(D^{\mu}[a] \mathscr{J}_{\mu}^{(c o v)}\right)(x)=-\delta J \equiv \mathscr{A}[\omega, a, \theta]_{c o v},
$$

where

$$
\begin{aligned}
& \delta J=d \bar{\psi}^{\prime} d \psi^{\prime}-d \bar{\psi} d \psi \quad \psi^{\prime}=\psi+i \omega P_{L} \psi, \bar{\psi}^{\prime}=\bar{\psi}-i \bar{\psi} P_{R} \omega \\
& \delta J=\lim _{\Lambda \rightarrow \infty} \int d^{4} x \sum_{m}\left\{\phi_{m}^{\dagger} \omega e^{-\lambda_{m}^{2} / \Lambda^{2}} P_{R} \phi_{m}-\varphi_{m}^{\dagger} \omega e^{-\lambda_{m}^{2} / \Lambda^{2}} P_{L} \varphi_{m}\right\} \\
& \mathscr{J}_{\mu}^{a,(c o v)}(x)=\frac{1}{Z[a, \theta]} \int d \bar{\psi} d \psi \frac{\delta S_{\text {fermionic }}}{\delta a_{\mu}^{a}(x)} e^{-S_{\text {fermionic }}, S_{\text {fermionic }}=\int d^{4} x \bar{\psi} i \mathscr{D} \psi .}
\end{aligned}
$$

By changing to a plane wave basis, one gets

$$
\begin{aligned}
& \mathscr{A}[\omega, a, \theta]_{c o v}=\lim _{\Lambda \rightarrow \infty}-\int d^{4} x \operatorname{Tr} \omega(x) \int \frac{d^{4} p}{(2 \pi)^{4}} \operatorname{tr}\left\{\left(\gamma_{5} e^{-i p x} e^{-\frac{\left.\left(i D^{(\theta)}\right)(a)\right)^{2}}{\Lambda^{2}}} e^{i p x}\right)\right\}, \\
& \not^{(\theta)}(a)=\not D+R, R=-\left[\frac{1}{4} \theta^{\alpha \beta} f_{\alpha \beta} \gamma^{\mu} D_{\mu}+\frac{1}{2} \theta^{\alpha \beta} \gamma^{\rho} f_{\rho \alpha} D_{\beta}\right] .
\end{aligned}
$$

Let us expand next the previous result in powers of $\theta$ and remove the terms that vanish as $\Lambda \rightarrow \infty$. Thus one gets

$$
\begin{aligned}
& \mathscr{A}[\omega, a, \theta]_{\text {cov }}=\lim _{\Lambda \rightarrow \infty}-\int d^{4} x \operatorname{Tr} \omega \int \frac{d^{4} p}{(2 \pi)^{4}} \operatorname{tr}\left\{\left(\gamma_{5} e^{-i p x} e^{-\frac{\left(i D^{(\theta)}(a)\right)^{2}}{\Lambda^{2}}} e^{i p x}\right)\right\}= \\
& \mathscr{A}^{(\text {ordinary })}[\omega, a]+\mathscr{A}^{(1)}[\omega, a, \theta]+o\left(\theta^{2}\right) \\
& \mathscr{A}[\omega, a]^{(\text {ordinary })}=-\frac{1}{32 \pi^{2}} \int d^{4} x \operatorname{Tr} \omega \varepsilon^{\mu v \rho \sigma} f_{\mu v} f_{\rho \sigma}, \\
& \mathscr{A}^{(1)}[\omega, a, \theta]=\int d^{4} x \operatorname{Tr} \omega(x)\left[\mathscr{A}_{1}(x)+\mathscr{A}_{2}(x)+\mathscr{A}_{3}(x)\right]
\end{aligned}
$$




$$
\begin{aligned}
& \mathscr{A}_{1}=-\sum_{l=0}^{1} \lim _{\Lambda \rightarrow \infty} 2 i \int \frac{d^{4} q}{(2 \pi)^{4}} e^{-q^{2}} \frac{1}{2} \operatorname{tr} \gamma_{5} \not D^{2 l}(\Lambda q)\{\not D(\Lambda q), R(\Lambda q)\} \not D^{2(1-l)}(\Lambda q) \mathrm{II}, \\
& \mathscr{A}_{2}=-\sum_{l=0}^{2} \lim _{\Lambda \rightarrow \infty} 2 i \int \frac{d^{4} q}{(2 \pi)^{4}} e^{-q^{2}} \frac{1}{3 ! \Lambda^{2}} \operatorname{tr} \gamma_{5} \not D^{2 l}(\Lambda q)\{\not D(\Lambda q), R(\Lambda q)\} \not D^{2(2-l)}(\Lambda q) \mathrm{II}, \\
& \mathscr{A}_{3}=-\sum_{l=0}^{3} \lim _{\Lambda \rightarrow \infty} 2 i \int \frac{d^{4} q}{(2 \pi)^{4}} e^{-q^{2}} \frac{1}{4 ! \Lambda^{4}} \operatorname{tr} \gamma_{5} \not D^{2 l}(\Lambda q)\{\not D(\Lambda q), R(\Lambda q)\} \not D^{2(3-l)}(\Lambda q) \mathrm{II} .
\end{aligned}
$$

Some lengthy algebra and the fact that the $a_{\mu}$ 's commute $-\mathrm{U}(1)$ case- lead to

$$
\begin{aligned}
& \mathscr{A}_{1}=-\frac{i}{8 \pi^{2}} \theta^{\alpha \beta} \varepsilon^{\mu \nu \rho \sigma}\left(-\frac{1}{2} f_{\alpha \beta} f_{\mu v} f_{\rho \sigma}-f_{v \alpha} f_{\mu \beta} f_{\rho \sigma}\right) \\
& +\frac{1}{16 \pi^{2}} \theta^{\alpha \beta} \varepsilon^{\mu v \rho \sigma}\left[f_{\mu v}\left(\partial_{\rho} f_{\sigma \alpha} D_{\beta} I+\frac{1}{2} \partial_{\rho} f_{\alpha \beta} D_{\sigma} I\right)+\partial_{\mu} f_{v \alpha} f_{\rho \sigma} D_{\beta} \Pi\right. \\
& \left.+\frac{1}{2} \partial_{\mu} f_{\alpha \beta} f_{\rho \sigma} D_{v} \mathrm{II}\right] \\
& \mathscr{A}_{2}=-\frac{i}{2(4 \pi)^{2}} \theta^{\alpha \beta} \varepsilon^{\mu v \rho \sigma} f_{\alpha \beta} f_{\mu v} f_{\rho \sigma}+ \\
& -\frac{1}{16 \pi^{2}} \theta^{\alpha \beta} \varepsilon^{\mu v \rho \sigma}\left[f_{\mu v} \partial_{\rho} f_{\sigma \alpha} D_{\beta} I I+\partial_{\mu} f_{v \alpha} f_{\rho \sigma} D_{\beta} I I+\frac{1}{2}\left(f_{\mu v} \partial_{\rho} f_{\alpha \beta} D_{\sigma} I\right.\right. \\
& \left.\left.+\partial_{\mu} f_{\alpha \beta} f_{\rho \sigma} D_{v} \mathrm{II}\right)\right] \\
& \mathscr{A}_{3}=0 \text {. }
\end{aligned}
$$

So, finally the first order in $\theta$ correction to the ordinary anomaly vanishes:

$$
\begin{aligned}
& \mathscr{A}^{(1)}[\omega, a, \theta]=\int d^{4} x \operatorname{Tr} \omega(x)\left[\mathscr{A}_{1}(x)+\mathscr{A}_{2}(x)+\mathscr{A}_{3}(x)\right]= \\
& \frac{i}{32 \pi^{2}} \theta^{\alpha \beta} \varepsilon^{\mu v \rho \sigma} \int d^{4} x \operatorname{Tr} \omega\left(f_{\alpha \beta} f_{\mu v} f_{\rho \sigma}+4 f_{v \alpha} f_{\mu \beta} f_{\rho \sigma}\right)=0, \\
& \mathscr{A}[\omega, a, \theta]_{\text {cov }}=\mathscr{A}^{\text {(ordinary) }}[\omega, a]+o\left(\theta^{2}\right) .
\end{aligned}
$$

This shows complete agreement with the result obtained by using Dimensional Regularization. Higher order corrections in $\theta$ and the nonabelian case are still to be worked out.

\section{Renormalisability}

The issue of the renormalisability of noncommutative gauge theories formulated within the enveloping-algebra formalism started off splendidly, for it was shown by Bichl, Grimstrup, Grosse, Popp. Schweda and Wulkenhaar [11] that the photon two-point function is renormalisable at any order in $\theta$. Unfortunately, Wulkenhaar showed [12] that this $\theta$-expanded QED was not renormalisable mainly due to the infamous four-point fermionic divergence:

$$
\frac{c}{D-4} \theta^{\alpha \beta} \varepsilon_{\mu v \rho \sigma} \int d^{4} x \bar{\psi} \gamma_{5} \gamma^{\rho} \psi \bar{\psi} \gamma^{\sigma} \psi
$$

Four years after Wulkenhaar's paper, there came along the encouraging results by Buric, Latas and Radovanovic [13], and, Buric, Radovanovic and Trampetic [14], that the gauge sector of SU(N) and the noncommutative Standard Model were one-loop renormalisable at first order in $\theta$. And yet, due to the infamous four-point fermionic divergence above, the construction of theories with a renormalisable one-loop and first-order-in- $\theta$ matter sector remained an open issue. Then it appeared a new paper by Buric, Latas, Radovanovic and Trampetic [15], where they showed that the divergence of the four-point fermionic function vanishes for a noncommutative $S U(2)$ chiral theory with the matter sector being an SU(2)-doublet of noncommutative left-handed fermions. This result was later generalized in ref. [16] to any noncommutative GUT-inspired theory with only fermions as matter fields. Let me recall that by noncommutative GUT-inspired theories I mean 
gauge theories whose noncommutative fermions are all -this is capital- left-handed multiplets, which transforms under a Grand Unification group. Thus, one of the obstacles -what about the renormalisability of the other 1PI functions?- to achieve one-loop and first-order-in- $\theta$ renormalisability had been removed by selecting Grand Unification -and, as we shall see, family unification, besides- as a guiding principle.

The absence of the infamous four-point fermionic divergence opened up the possibility of building noncommutative theories with massless fermionic noncommutative chiral matter that are one-loop renormalisable at first order in $\theta$. Actually, Wulkenhaar had already pointed out in ref. [12] that, in the massless case, noncommutative QED is (off-shell) one-loop renormmalisable at first order in $\theta$, if one forgets about the fermionic four-point function. At long last, it was shown in ref. [17] that noncommutative GUT-inspired theories, with a matter sector made out of fermions and no scalars, were, on-shell and at first order in $\theta$, one-loop-renormalisable for any anomaly safe compact simple gauge group, if, and only if, all the flavour fermionic multiplets carry irreps with the same quadratic Casimir, ie, renormalisability is very partial to family unification. This selects $\mathrm{SO}(10), \mathrm{E}_{6}$, and drops $\mathrm{SU}(5)$, as noncommutative Grand Unification groups-see [18].

We shall close this section with a quick recap of the results in ref. [17]. The action of the noncommutative GUT-inspired models in ref. [17] reads

$$
\begin{aligned}
& S=\int d^{4} x-\frac{1}{2 g^{2}} \operatorname{Tr} F_{\mu v} \star F^{\mu v}+\bar{\Psi}_{L} i \not D \Psi_{L}, \\
& F_{\mu v}=\partial_{\mu} A_{v}-\partial_{v} A_{\mu}-i\left[A_{\mu}, A_{v}\right]_{\star}, \quad D_{\mu} \psi_{L}=\partial_{\mu} \Psi_{L}-i \rho_{\Psi}\left(A_{\mu}\right) \star \Psi_{L},
\end{aligned}
$$

$\rho_{\psi}$ denotes an arbitrary unitary representation, which is a direct sum of irreducible representations, $\rho_{\psi}=\bigoplus_{r=1}^{F} \rho_{\psi}^{r}$. Then, lengthy computations led to the following result:

Once $\psi_{L}^{r}, g$ and $\theta$ have been renormalised as follows

$$
\begin{aligned}
& \psi^{r}=\left(Z_{\psi}^{r}\right)^{1 / 2} \psi_{R}^{r}, g=\mu^{-\varepsilon} Z_{g} g_{R}, \theta^{\mu v}=Z_{\theta} \theta_{R}^{\mu v}, \\
& Z_{\psi}^{r}=1+\frac{g^{2} C_{2}(r)}{16 \pi^{2} \varepsilon}, Z_{g}=1+\frac{g^{2}}{16 \pi^{2} \varepsilon}\left[\frac{11}{6} C_{2}(G)-\frac{2}{3} \sum_{r} c_{2}(r)\right], \\
& Z_{\theta}=-Z_{\psi}^{r}-\frac{g^{2}}{48 \pi^{2} \varepsilon}\left(13 C_{2}(r)-4 C_{2}(G)\right),
\end{aligned}
$$

the UV divergences, at one-loop and first order in $\theta$, which remain in the background-field effective action are given by the on-shell vanishing expression

$$
S^{\mathrm{ct}}=\int d^{4} x \frac{\delta S}{\delta a_{\mu}^{a}(x)} F_{\mu}^{a}[a, \psi]+\left(\sum_{r} \frac{\delta S}{\delta \psi^{r}(x)} G_{r}[a, \psi]+\text { c.c. }\right),
$$

where

$$
\begin{aligned}
F_{\mu}= & y_{1} \theta^{\alpha \beta} \mathscr{D}_{\mu} f_{\alpha \beta}+y_{2} \theta_{\mu}{ }^{\alpha} D^{v} f_{v \alpha}+\sum_{r} y_{3}^{r} \theta_{\mu}^{\alpha}\left(\bar{\psi}_{r} \gamma_{\alpha} P_{L} T^{a} \psi^{r}\right) T^{a} \\
& +i \sum_{r} y_{4}^{r} \theta^{\alpha \beta}\left(\bar{\psi}_{r} \gamma_{\mu \alpha \beta} P_{L} T^{a} \psi^{r}\right) T^{a}+y_{5} \tilde{\theta}_{\mu}^{\beta} D^{v} f_{v \beta}, \\
G_{r, L}= & k_{1}^{r} \theta^{\alpha \beta} f_{\alpha \beta} P_{L} \psi^{r}+k_{r}^{2} \theta^{\alpha \beta} \gamma_{\alpha \mu} P_{L} f_{\beta}{ }^{\mu} \psi^{r} \\
& +k_{3}^{r} \theta^{\alpha \beta} \gamma_{\alpha \mu} P_{L} D_{\beta} D^{\mu} \psi^{r}+k_{4}^{r} \theta^{\alpha \beta} \gamma_{\alpha \beta} P_{L} D^{2} \psi^{r} \\
& +k_{5}^{r} \tilde{\theta}^{\alpha \beta} \gamma_{5} P_{L} f_{\alpha \beta} \psi^{r} ; y_{i} \in \mathbb{R}, k_{i} \in \mathbb{C},
\end{aligned}
$$


with

$$
\begin{aligned}
& y_{1}=\operatorname{Im} k_{1}^{r}, y_{3}^{r}=2 g^{2} y_{2}, \\
& y_{4}^{r}=-y_{5} g^{2}-\frac{g^{4}}{384 \pi^{2}}\left(16 C_{2}(r)-13 C_{2}(G)\right), \\
& \operatorname{Re} k_{1}^{r}=-\frac{1}{2} \operatorname{Im} k_{3}^{r}-\frac{g^{2}}{384 \pi^{2} \varepsilon}\left(13 C_{2}(r)-8 C_{2}(G)\right), \\
& \operatorname{Im} k_{5}^{r}=-\frac{g^{2}}{384 \pi^{2} \varepsilon}\left(11 C_{2}(r)-8 C_{2}(G)\right), \\
& \operatorname{Im} k_{4}^{r}=\frac{g^{2} C_{2}(r)}{384 \pi^{2} \varepsilon}, \operatorname{Re} k_{2}^{r}=-\frac{5 g^{2}}{192 \pi^{2} \varepsilon}\left(2 C_{2}(r)-C_{2}(G)\right), \\
& \operatorname{Im} k_{2}^{r}=\operatorname{Re} k_{3}^{r}=2 \operatorname{Re} k_{5}^{r}=-2 \operatorname{Re} k_{4}^{r} .
\end{aligned}
$$

Let me stress that $y_{1}, y_{2}, y_{5}$ and $Z_{\theta}$ above must be flavour independent, and so must be $y_{3}, y_{4}$. Hence. $C_{2}(r)$, must be the same for all irreps, which in turn demands family unification.

\section{Yukawa Terms in Noncommutative GUTs}

Here I shall address the issue of constructing Yukawa terms in noncommutative SO(10) and $\mathrm{E}_{6}$ GUTs. For details I refer the reader to ref. [19].

Let us recall that Yukawa terms of ordinary $\mathrm{SO}(10)$ and $\mathrm{E}_{6}$ read

$$
\mathscr{Y}^{\text {(ord) }}=\int d^{4} x y_{f f^{\prime}} \mathscr{C}_{A i B} \widetilde{\psi}_{A f}^{\alpha} \psi_{\alpha B f^{\prime}} \phi_{i},
$$

where $\widetilde{\psi}_{f}^{\alpha} \equiv\left(\psi_{f}^{\alpha}\right)^{t}$, and $\gamma_{f f^{\prime}}$ denotes the Yukawa coefficients. For $\mathrm{SO}(10)$, each fermionic multiplet $\psi_{\alpha f^{\prime}}$ carry the 16 irrep of $\mathrm{SO}(10)$, whereas, in the $\mathrm{E}_{6} \mathrm{GUT}, \psi_{\alpha f^{\prime}}$ transforms under the 27 irrep of $\mathrm{E}_{6}$. The Higgs multiplets in $\mathrm{SO}(10)$ carry any of the following irrreps: 10,120 and $\overline{126}$. In the $\mathrm{E}_{6}$ case the Higgs multiplets furnish any of the irreps of $E_{6}$ that I enumerate now: 27, 351' and 351. Indeed, one has the following Clebsch-Gordan decompositions

$$
16 \bigotimes 16=(10 \bigoplus 126)_{\mathrm{s}} \bigoplus 120_{\mathrm{as}}, 27 \bigotimes 27=\left(\overline{27} \bigoplus \overline{351^{\prime}}\right)_{\mathrm{s}} \bigoplus \overline{351}_{\mathrm{as}}
$$

In eq. (4.1), $\mathscr{C}_{A i B}$ is an invariant tensor:

$$
\widetilde{\Sigma}_{A C}^{a} \mathscr{C}_{C i B}+\mathscr{C}_{A j B} M_{j i}^{a}+\mathscr{C}_{A j C} \Sigma_{C B}^{a}=0,
$$

where $\widetilde{\Sigma}^{a}, M^{a}$ and $\Sigma^{a}$ denote the group generators in the irreps furnished by $\widetilde{\psi}_{A f}^{\alpha}, \phi_{i}$ and $\psi_{B f^{\prime}}^{\alpha}$, respectively.

Let $\widetilde{\Psi}_{A f}^{\alpha}, \Psi_{\alpha B f^{\prime}}$ and $\Phi_{i}$ denote the noncommutative fermionic and Higgs fields defined by standard Seiberg-Witten maps, ie, solutions to

$$
s_{N C}(\text { NCField }) \equiv i \Lambda \star(\text { NCField })=s(\text { NCField })
$$

that match the ordinary fields at $\theta=0$. Then, a naive noncommutative version

$$
\mathscr{Y}_{(\text {naive })}^{(N C)}=\int d^{4} x y_{f f^{\prime}} \mathscr{C}_{A i B} \widetilde{\Psi}_{A f}^{\alpha} \star \Psi_{\alpha B f^{\prime}} \star \Phi_{i}
$$

of the ordinary Yukawa term in eq. (4.1) would not do! Indeed,

$$
\begin{aligned}
& 0 \neq s_{N C} \mathscr{Y}_{(\text {naive })}^{(N C)}= \\
& \begin{aligned}
& \int d^{4} x y_{f f^{\prime}} \mathscr{C}_{A i B}\left(i \widetilde{\Lambda}_{A C} \star \widetilde{\Psi}_{C f}^{\alpha} \star \Psi_{\alpha B f^{\prime}} \star \Phi_{i}+\widetilde{\Psi}_{A f}^{\alpha} \star i \Lambda_{B C} \star \Psi_{\alpha C f^{\prime}} \star \Phi_{i}\right. \\
&+\left.\widetilde{\Psi}_{A f}^{\alpha} \star \Psi_{\alpha B f^{\prime}} \star i \Lambda_{i j} \star \Phi_{j}\right),
\end{aligned}
\end{aligned}
$$


for the $\star$-product is not commutative and $\mathscr{C}_{A i B}$ is not invariant for enveloping-algebra valued $\Lambda$ 's.

I shall now explain my strategy for constructing noncommutative Yukawa terms. To carry over the properties of $\mathscr{C}_{A i B}$ to the noncommutative theory in a consistent way, one first combines $\mathscr{C}_{A i B}$ with the ordinary fields $\widetilde{\psi}_{A f}^{\alpha}, \psi_{\alpha B f^{\prime}}$ and $\phi_{i}$, and, then, defines new ordinary fields that transform under tensor products of ordinary irreps of the gauge group, but have the very same number of freedom as $\widetilde{\psi}_{A f}^{\alpha}, \psi_{\alpha B f^{\prime}}$ and $\phi_{i}$ :

$$
\phi_{A B}=\mathscr{C}_{A i B} \phi_{i}, \quad \widetilde{\psi}_{i B f}^{\alpha}=\widetilde{\psi}_{A f}^{\alpha} \mathscr{C}_{A i B}, \quad \psi_{\alpha A i f^{\prime}}=\mathscr{C}_{A i B} \psi_{\alpha B i f^{\prime}}
$$

The BRS transformations of these new fields run thus:

$$
\begin{aligned}
& s \phi_{A B}=-i \widetilde{\lambda}_{A C}^{(\psi)} \phi_{C B}-i \phi_{A C} \lambda_{C B}^{(\psi)} \\
& s \widetilde{\psi}_{i B f}^{\alpha}=-i \widetilde{\lambda}_{i j}^{(\phi)} \widetilde{\psi}_{j B f}^{\alpha}-i \widetilde{\psi}_{i C f}^{\alpha} \lambda_{C B}^{(\psi)}, \\
& s \psi_{\alpha A i f^{\prime}}=-i \widetilde{\lambda}_{A C}^{(\psi)} \psi_{\alpha C i f^{\prime}}-i \psi_{\alpha A j f^{\prime}} \lambda_{j i}^{(\phi)}
\end{aligned}
$$

Next, to each ordinary field $\phi_{A B}, \widetilde{\psi}_{i B f}^{\alpha}$ and $\psi_{\alpha A i f^{\prime}}$, one associates a noncommutative counterpart

$$
\Phi_{A B}\left[\phi_{A B}, a_{\mu}^{a}, \theta\right], \quad \widetilde{\Psi}_{i B f}^{\alpha}\left[\widetilde{\psi}_{i B f}^{\alpha}, a_{\mu}^{a}, \theta\right] \quad \text { and } \quad \Psi_{\alpha A i f^{\prime}}\left[\psi_{\alpha A i f^{\prime}}, a_{\mu}^{a}, \theta\right]
$$

which, respectively, are solutions to the following Hybrid Seiberg-Witten map equations:

$$
s_{N C} \Phi_{A B}=s \Phi_{A B}, \quad s_{N C} \widetilde{\Psi}_{i B f}^{\alpha}=s \widetilde{\Psi}_{i B f}^{\alpha}, \quad s_{N C} \Psi_{\alpha A i f^{\prime}}=s \Psi_{\alpha A i f^{\prime}},
$$

where one defines

$$
\begin{aligned}
& s_{N C} \Phi_{A B} \equiv-i \widetilde{\Lambda}_{A C}^{(\psi)} \star \Phi_{C B}-i \Phi_{A C} \star \Lambda_{C B}^{(\psi)}, \\
& s_{N C} \widetilde{\Psi}_{i B f}^{\alpha} \equiv-i \widetilde{\Lambda}_{i j}^{(\phi)} \star \widetilde{\Psi}_{j B f}^{\alpha}-i \widetilde{\Psi}_{i C f}^{\alpha} \star \Lambda_{C B}^{(\psi)} \\
& s_{\mathrm{nc}} \Psi_{\alpha A i f^{\prime}} \equiv-i \widetilde{\Lambda}_{A C}^{(\psi)} \star \Psi_{\alpha C i f^{\prime}}-i \Psi_{\alpha A j f^{\prime}} \star \Lambda_{j i}^{(\phi)} .
\end{aligned}
$$

Let me point out that the action from the left and from the right (as opposed to both actions from the left or both from the right) of the $\Lambda$ 's is the only choice consistent with $\left(s_{N C}\right)^{2}=0$ !. The solutions to the equations in eq. (4.2) are Seiberg-Witten maps of hybrid type, a notion introduced by Schupp [7].

We are now in the position to obtain in a natural (naive) way noncommutative $\mathrm{SO}(10), \mathrm{E}_{6}$ Yukawa terms from their ordinary counterparts. Indeed, in terms of $\phi_{A B}$, the ordinary Yukawa term reads:

$$
\mathscr{Y}_{1}^{(\text {ord })} \equiv \mathscr{Y}^{(\text {ord })}=\int d^{4} x \gamma_{f f^{\prime}} \widetilde{\psi}_{A f}^{\alpha} \phi_{A B} \psi_{\alpha B f^{\prime}},
$$

so that, its noncommutative counterpart is

$$
\mathscr{Y}_{1}^{(\mathrm{nc})}=\int d^{4} x y_{f f^{\prime}}^{(1)} \widetilde{\Psi}_{A f}^{\alpha} \star \Phi_{A B} \star \Psi_{\alpha B f^{\prime}}
$$

In words: the noncommutative Yukawa term associated to $\mathscr{Y}_{1}^{(\text {ord })}$ is obtained by replacing each ordinary field in the latter with its noncommutative counterpart and the ordinary product with the $\star$-product. 
By construction $\mathscr{Y}_{1}^{(\mathrm{nc})}$ is invariant under the following noncommutative BRS transformations:

$$
\begin{aligned}
& s_{N C} \widetilde{\Psi}_{A f}^{\alpha}=i \widetilde{\Psi}_{B f}^{\alpha} \star \widetilde{\Lambda}_{B A}^{(\psi)}, \quad s_{\mathrm{nc}} \Psi_{\alpha B f^{\prime}}=i \Lambda_{B C}^{(\psi)} \star \Psi_{\alpha C f^{\prime}}, \\
& s_{N C} \Phi_{A B}=-i \widetilde{\Lambda}_{A C}^{(\psi)} \star \Phi_{C B}-i \Phi_{A C} \star \Lambda_{C B}^{(\psi)}, \\
& s_{N C} \widetilde{\Lambda}_{B A}^{(\psi)}=-i \widetilde{\Lambda}_{B C}^{(\psi)} \star \widetilde{\Lambda}_{C A}^{(\psi)}, \quad s_{\mathrm{nc}} \Lambda_{B C}^{(\psi)}=i \Lambda_{B D}^{(\psi)} \star \Lambda_{D C}^{(\psi)} .
\end{aligned}
$$

The Seiberg-Witten maps which define the noncommutative fields are

$$
\begin{aligned}
\widetilde{\Psi}_{A f}^{\alpha}= & \widetilde{\psi}_{A f}^{\alpha}-\frac{1}{2} \theta^{\mu v} \partial_{\mu} \widetilde{\psi}_{B f}^{\alpha} \widetilde{a}_{v B A}^{(\psi)}+\frac{i}{4} \theta^{\mu v} \widetilde{\psi}_{C f}^{\alpha} \widetilde{a}_{\mu C B}^{(\psi)} \widetilde{a}_{v B A}^{(\psi)}+O\left(\theta^{2}\right), \\
\Phi_{A B}=\phi_{A B} & +\frac{1}{2} \theta^{\mu v} \widetilde{a}_{\mu A C}^{(\psi)} \partial_{v} \phi_{C B}+\frac{i}{4} \theta^{\mu v} \widetilde{a}_{\mu A C}^{(\psi)} \widetilde{a}_{v C D}^{(\psi)} \phi_{D B}+ \\
& \quad+\frac{1}{2} \theta^{\mu v} \partial_{\mu} \phi_{A C} a_{v C B}^{(\psi)}+\frac{i}{4} \theta^{\mu v} \phi_{A C} a_{\mu C D}^{(\psi)} a_{v D B}^{(\psi)} \\
& \quad+\frac{i}{2} \theta^{\mu v} \widetilde{a}_{\mu A C}^{(\psi)} \phi_{C D} a_{v D B}^{(\psi)}+O\left(\theta^{2}\right),
\end{aligned}
$$

Let me now point out that if we use $\widetilde{\psi}_{i B f}^{\alpha}$ and $\psi_{\alpha A i f^{\prime}}$ to formulate an ordinary Yukawa term, we obtain the same ordinary Yukawa term:

$$
\begin{aligned}
& \mathscr{Y}_{2}^{\text {(ord) }}=\int d^{4} x \mathscr{y}_{f f^{\prime}} \widetilde{\phi}_{i} \widetilde{\psi}_{i B f}^{\alpha} \psi_{\alpha B f^{\prime}}, \\
& \mathscr{Y}_{3}^{\text {(ord) }}=\int d^{4} x \mathscr{Y}_{f f^{\prime}} \widetilde{\psi}_{A f}^{\alpha} \psi_{\alpha A i f^{\prime}} \phi_{i}, \\
& \mathscr{Y}_{1}^{\text {(ord) }}=\mathscr{Y}_{2}^{\text {(ord) }}=\mathscr{Y}_{3}^{\text {(ord) }} .
\end{aligned}
$$

And yet, the noncommutative counterparts of $\mathscr{Y}_{2}^{(\text {ord })}$ and $\mathscr{Y}_{3}^{(\text {ord })}$ are not equal:

$$
\begin{aligned}
& \mathscr{Y}_{2}^{(\mathrm{nc})}=\int d^{4} x Y_{f f^{\prime}}^{(2)} \widetilde{\Phi}_{i} \star \widetilde{\Psi}_{i B f}^{\alpha} \star \Psi_{\alpha B f^{\prime}} \\
& \mathscr{Y}_{3}^{(\mathrm{nc})}=\int d^{4} x \mathscr{Y}_{f f^{\prime}}^{(3)} \mathscr{C}_{A i B} \widetilde{\Psi}_{A f}^{\alpha} \phi_{i} \psi_{\alpha B f^{\prime}} \\
& \mathscr{Y}_{1}^{(\mathrm{nc})} \neq \mathscr{Y}_{2}^{(\mathrm{nc})} \neq \mathscr{Y}_{3}^{(\mathrm{nc})} \neq \mathscr{Y}_{1}^{(\mathrm{nc})}
\end{aligned}
$$

Hence, I propose the following noncommutative Yukawa term

$$
\mathscr{Y}^{(\mathrm{nc})}=\mathscr{Y}_{1}^{(\mathrm{nc})}+\mathscr{Y}_{2}^{(\mathrm{nc})}+\mathscr{Y}_{3}^{(\mathrm{nc})} .
$$

It can be shown -see ref [19]- that at first order in $\theta$ this is the most general BRS invariant Yukawatype term

$$
\theta^{\mu \nu} \int d^{4} x \mathscr{Y}_{f f^{\prime}} \psi_{A f}^{\alpha} \mathscr{V}_{\mu \nu}^{A i B}\left[\theta^{\rho \sigma}, \partial_{\mu}, a_{v}^{a}\right] \phi_{i} \psi_{\alpha B f^{\prime}}
$$

that one can write. This Yukawa term is therefore renormalisable at first order in $\theta$.

\section{What about SUSY?}

For $U(N)$ in the fundamental rep., $\mathscr{N}=1 \mathrm{SYM}$ exists in the enveloping-algebra formalism as a classical theory:

$$
S_{N C S Y M}=\frac{1}{2 g^{2}} \operatorname{Tr} \int d^{4} x\left[-\frac{1}{2} F^{\mu v} \star F_{\mu v}-2 i \Lambda^{\alpha} \star \sigma_{\alpha \dot{\alpha}}^{\mu} D_{\mu} \bar{\Lambda}^{\dot{\alpha}}+D \star D\right]
$$


where

$$
A_{\mu}=A_{\mu}\left[a, \lambda_{\alpha}, d, \theta\right], \Lambda_{\alpha}\left[a, \lambda_{\alpha}, d, \theta\right] \text { and } D=D\left[a, \lambda_{\alpha}, d, \theta\right]
$$

are SW maps. $S_{N C S Y M}$ is invariant under $\mathscr{N}=1$ SUSY:

- linearly realized in terms of the noncommutative fields ( there is a local superfield formulation)

and

- nonlinearly realized in terms of the ordinary fields (no local superfield formulation exists, but a nonlocal one does, at least for U(1) -see [20]).

The $\mathscr{N}=1$ SUSY transformations of the noncommutative fields read

$$
\begin{aligned}
& A_{\mu}[\varphi, \theta] \rightarrow A_{\mu}^{(\varepsilon)}[\varphi, \theta]=A_{\mu}[\varphi, \theta]+\delta_{\varepsilon} A_{\mu}[\varphi, \theta] \\
& \Lambda_{\alpha}[\varphi, \theta] \rightarrow \Lambda_{\alpha}^{(\varepsilon)}[\varphi, \theta]=\Lambda_{\alpha}[\varphi, \theta]+\delta_{\varepsilon} \Lambda_{\alpha}[\varphi, \theta] \\
& D[\varphi, \theta] \rightarrow D^{(\varepsilon)}[\varphi, \theta]=D[\varphi, \theta]+\delta_{\varepsilon} D[\varphi, \theta]
\end{aligned}
$$

where $\varphi$ denotes generically the ordinary fields and

$$
\begin{aligned}
& \delta_{\varepsilon} A^{\mu}=i \varepsilon^{\alpha} \sigma_{\alpha \dot{\alpha}}^{\mu} \bar{\Lambda}^{\dot{\alpha}}+i \bar{\varepsilon}^{\dot{\alpha}} \bar{\sigma}_{\dot{\alpha} \alpha}^{\mu} \Lambda^{\alpha}, \\
& \delta_{\varepsilon} \Lambda_{\alpha}=\left(\sigma^{\mu v}\right)_{\alpha}{ }^{\beta} \varepsilon_{\beta} F_{\mu v}+i \varepsilon_{\alpha} D, \\
& \delta_{\varepsilon} D=-\varepsilon^{\alpha} \sigma_{\alpha \dot{\alpha}}^{\mu} D_{\mu} \bar{\Lambda}^{\dot{\alpha}}+\bar{\varepsilon}^{\dot{\alpha}} \bar{\sigma}_{\dot{\alpha} \alpha}^{\mu} D_{\mu} \Lambda^{\alpha} .
\end{aligned}
$$

Now, the SUSY transformations have just introduced -do not forget that we are in the $U(N)$ case in the fundamental representation - can be induced by performing a nonlinear variation of the ordinary fields, which up to first order in $\theta$, reads

$$
\begin{aligned}
\delta_{\varepsilon} a_{\mu}= & \frac{1}{4} \varepsilon \sigma_{\mu} \bar{\lambda}-\frac{1}{4} \bar{\varepsilon} \bar{\sigma}_{\mu} \lambda+\frac{1}{16} \theta^{v \rho}\left[\left\{a_{v}, 2 D_{\rho}\left(\varepsilon \sigma_{\mu} \bar{\lambda}-\bar{\varepsilon} \bar{\sigma}_{\mu} \lambda\right)-i\left[a_{\rho}, \varepsilon \sigma_{\mu} \bar{\lambda}-\bar{\varepsilon} \bar{\sigma}_{\mu} \lambda\right]\right\}\right. \\
& -\left\{\varepsilon \sigma_{n} \bar{\lambda}-\bar{\varepsilon} \bar{\sigma}_{v} \lambda, \partial_{\rho} a_{\mu}+f_{\rho \mu}\right\}-\left\{a_{v}, \partial_{\rho}\left(\varepsilon \sigma_{\mu} \bar{\lambda}-\bar{\varepsilon} \bar{\sigma}_{\mu} \lambda\right)+D_{\rho}\left(\varepsilon \sigma_{\mu} \bar{\lambda}-\bar{\varepsilon} \bar{\sigma}_{m} \lambda\right)\right. \\
& \left.\left.-D_{\mu}\left(\varepsilon \sigma_{\rho} \bar{\lambda}-\bar{\varepsilon} \bar{\sigma}_{l} \lambda\right)\right\}\right]+\theta^{2}, \\
\delta_{\varepsilon} \lambda_{\alpha}= & -\varepsilon_{\alpha} d+2 i \varepsilon_{\gamma}\left(\sigma^{\mu v}\right)_{\alpha}^{\gamma} f_{\mu v}+\frac{1}{4} \theta^{v \rho}\left[-\frac{1}{4}\left\{\varepsilon \sigma_{v} \bar{\lambda}-\bar{\varepsilon} \bar{\sigma}_{v} \lambda, 2 D_{\rho} \lambda_{\alpha}-i\left[a_{\rho}, \lambda_{\alpha}\right]\right\}\right. \\
& \left.-\left\{a_{v}, 4 i D_{\rho}\left(\varepsilon_{\gamma}\left(\sigma^{\mu \rho}\right)_{\alpha}^{\gamma} f_{\mu \lambda}\right)+2\left[a_{\rho}, \varepsilon_{\gamma}\left(\sigma^{\mu \lambda}\right)_{\alpha}^{\gamma} f_{\mu \lambda}\right]+\frac{i}{4}\left[\varepsilon \sigma_{\rho} \bar{\lambda}-\bar{\varepsilon} \bar{\sigma}_{l} \lambda, \lambda_{\alpha}\right]\right\}\right] \\
& +\theta^{2} \\
\delta_{\varepsilon} d= & i \bar{\varepsilon} \bar{\sigma}^{\mu} D_{\mu} \lambda+i \varepsilon \sigma^{\mu} D_{\mu} \bar{\lambda}+\frac{1}{4} \theta^{v \rho}\left[2 i\left\{f_{\mu v}, \bar{\varepsilon} \bar{\sigma}^{\mu} D_{\rho} \lambda+\varepsilon \sigma^{\mu} D_{\rho} \bar{\lambda}\right\}\right. \\
& +i\left\{a_{v},\left(\partial_{\rho}+D_{\rho}\right)\left(\bar{\varepsilon} \bar{\sigma}^{\mu} D_{\mu} \lambda+\varepsilon \sigma^{\mu} D_{\mu} \bar{\lambda}\right)\right\}-\frac{1}{4}\left\{\varepsilon \sigma_{v} \bar{\lambda}-\bar{\varepsilon} \bar{\sigma}_{v} \lambda, 2 D_{\rho} d-i\left[a_{\rho}, d\right]\right\} \\
& -\left\{a_{v}, 2 D_{\rho}\left(i \bar{\varepsilon} \bar{\sigma}^{\mu} D_{\mu} \lambda+i \varepsilon \sigma^{\mu} D_{\mu} \bar{\lambda}\right)-i\left[a_{\rho}, i \bar{\varepsilon} \bar{\sigma}^{\mu} D_{\mu} \lambda+i \varepsilon \sigma^{\mu} D_{\mu} \bar{\lambda}\right]\right. \\
& \left.\left.+\frac{i}{4}\left[\varepsilon \sigma_{\rho} \bar{\lambda}-\bar{\varepsilon} \bar{\sigma}_{\rho} \lambda, d\right]\right\}\right]+\theta^{2} .
\end{aligned}
$$

The following comments concerning the nonlinear variations of the ordinary fields in eq. (5.1) are now in order:

- They are truly $\mathscr{N}=1$ SUSY transformations,

$$
\left[\delta_{\varepsilon_{2}}, \delta_{\varepsilon_{1}}\right](\text { fields })=i\left(\varepsilon_{2} \sigma^{\mu} \bar{\varepsilon}_{1}-\varepsilon_{1} \sigma^{\mu} \bar{\varepsilon}_{2}\right) \partial_{\mu}(\text { fields })+\text { gauge transformations },
$$

due to the fact that the noncommutative fields carry a linear realisation of $\mathscr{N}=1$ SUSY. This holds at any order in $\theta$-see ref. [20]. 
- $\delta_{\varepsilon} a_{\mu}, \delta_{\varepsilon} \lambda_{\alpha}$ and $\delta_{\varepsilon} d$ belong to the Lie algebra of the ordinary gauge group only for $\mathrm{U}(\mathrm{N})$ in the fundamental rep. and its siblings, i.e.,

- for an arbitrary Lie algebra they take values on the enveloping-algebra: they are not ordinary field variations which are also ordinary fields.

The question that one should ask next is whether we can have SUSY noncommutative GUTs. It is apparent that for simple gauge groups in any representation, it still makes sense to consider the theory defined by the action

$$
S=\frac{1}{2 g^{2}} \operatorname{Tr} \int d^{4} x\left[-\frac{1}{2} F^{\mu v} \star F_{\mu v}-2 i \Lambda^{\alpha} \star \sigma_{\alpha \dot{\alpha}}^{\mu} D_{\mu} \bar{\Lambda}^{\dot{\alpha}}+D \star D\right]
$$

where

$$
A_{\mu}=A_{\mu}\left[a, \lambda_{\alpha}, d, \theta\right], \Lambda_{\alpha}\left[a, \lambda_{\alpha}, d, \theta\right] \quad \text { and } \quad D=D\left[a, \lambda_{\alpha}, d, \theta\right]
$$

are Seiberg-Witten maps. This action looks like a SUSY invariant noncommutative action, for it is invariant under the following transformations

$$
\begin{aligned}
& A_{\mu}[\varphi, \theta] \rightarrow A_{\mu}^{(\varepsilon)}[\varphi, \theta]=A_{\mu}[\varphi, \theta]+\delta_{\varepsilon} A_{\mu}[\varphi, \theta,] \\
& \Lambda_{\alpha}[\varphi, \theta] \rightarrow \Lambda_{\alpha}^{(\varepsilon)}[\varphi, \theta]=\Lambda_{\alpha}[\varphi, \theta]+\delta_{\varepsilon} \Lambda_{\alpha}[\varphi, \theta], \\
& D[\varphi, \theta] \rightarrow D^{(\varepsilon)}[\varphi, \theta]=D[\varphi, \theta]+\delta_{\varepsilon} D[\varphi, \theta], \\
& \delta_{\varepsilon} A^{\mu}=i \varepsilon^{\alpha} \sigma_{\alpha \dot{\alpha}}^{\mu} \bar{\Lambda}^{\dot{\alpha}}+i \bar{\varepsilon}^{\dot{\alpha}} \bar{\sigma}_{\dot{\alpha} \alpha}^{\mu} \Lambda^{\alpha}, \\
& \delta_{\varepsilon} \Lambda_{\alpha}=\left(\sigma^{\mu v}\right)_{\alpha}^{\beta} \varepsilon_{\beta} F_{\mu v}+i \varepsilon_{\alpha} D, \\
& \delta_{\varepsilon} D=-\varepsilon^{\alpha} \sigma_{\alpha \dot{\alpha}}^{\mu} D_{\mu} \bar{\Lambda}^{\dot{\alpha}}+\bar{\varepsilon}^{\dot{\alpha}} \bar{\sigma}_{\dot{\alpha} \alpha}^{\mu} D_{\mu} \Lambda^{\alpha},
\end{aligned}
$$

and these transformations satisfy the $\mathscr{N}=1$ SUSY algebra commutation relationship

$$
\left[\delta_{\varepsilon_{2}}, \delta_{\varepsilon_{1}}\right](\text { NCfields })=i\left(\varepsilon_{2} \sigma^{\mu} \bar{\varepsilon}_{1}-\varepsilon_{1} \sigma^{\mu} \bar{\varepsilon}_{2}\right) \partial_{\mu}(\text { NCfields })+\text { NC gauge transformations }
$$

Notice that $\varphi$ denotes the ordinary fields $a_{\mu}, \lambda_{\alpha}$ and $d$, and NC stands for noncommutative. This all goes in the right direction, but there is a catch: $A_{\mu}^{(\varepsilon)}[\varphi, \theta], \Lambda_{\alpha}^{(\varepsilon)}[\varphi, \theta]$ and $D^{(\varepsilon)}[\varphi, \theta]$ are not Seiberg-Witten maps in the sense that there are no ordinary fields $\varphi^{(\varepsilon)}[\varphi, \partial, \theta]$,

$$
\varphi^{(\varepsilon)}[\varphi, \partial, \theta]=\varphi+\varepsilon^{\alpha} \phi_{\alpha}[\varphi, \partial, \theta]+\bar{\varepsilon}_{\dot{\alpha}} \bar{\varphi}^{\dot{\alpha}}[\varphi, \partial, \theta],
$$

such that

$A_{\mu}^{(\varepsilon)}[\varphi, \theta]=A_{\mu}\left[\varphi^{(\varepsilon)}[\varphi, \partial, \theta] ; \theta\right], \Lambda_{\alpha}^{(\varepsilon)}[\varphi, \theta]=\Lambda_{\alpha}\left[\varphi^{(\varepsilon)}[\varphi, \partial, \theta] ; \theta\right], D^{(\varepsilon)}[\varphi, \theta]=D\left[\varphi^{(\varepsilon)}[\varphi, \partial, \theta] ; \theta\right]$,

where $A_{\mu}[\varphi ; \theta], \Lambda_{\alpha}[\varphi ; \theta]$ and $D[\varphi ; \theta]$ are solutions to the Seiberg-Witten equations satisfying $A_{\mu}[\varphi ; \theta=0]=a_{\mu}, \Lambda_{\alpha}[\varphi ; \theta=0]=\lambda_{\alpha}$ and $D[\varphi ; \theta=0]=d$. The transformations in eq. (5.2) are therefore defined from the space of noncommutative "physical" fields -those defined by the Seiberg-Witten map as explained above - into the space of general fields taking values on the enveloping algebra. The so remaining question is whether this invariance has any physical consequences. In this regard, it is worth noticing that -unlike in the U(N) case- the SUSY noncommutative $\mathrm{SU}(\mathrm{N})$ theory thus obtained is one-loop and first-order-in- $\theta$ (off-shell) renormalisable. This would be just a lucky chance unless there is a symmetry at work, at first order in $\theta$, that relates the gluon and gluino dynamics -see [21].

Some additional information regarding noncommutative SUSY theories defined by means of the Seiberg-Witten map can be found in refs. [22 -24] and [25]. 


\section{Open problems}

We shall conclude with a list of pressing problems:

- For $\mathrm{SO}(10)$ and $\mathrm{E}_{6}$, inclusion of a phenomenologically relevant noncommutative Higgs potential: a non trivial issue as implied by the construction of Yukawa terms.

- Study of the one-loop renormalisability of those noncommutative GUTS at first order in $\theta$.

- Construction and analysis of the properties of noncommutative SUSY SO(10), $\mathrm{E}_{6}$.

- Study of the phenomenological implications of noncommutative $\mathrm{SO}(10), \mathrm{E}_{6}$ GUTs.

- Gauge anomalies, Fujikawa's method and index theorems. Recall that the index theorem in $2 n+2$ dimensions gives the gauge anomaly in $2 n$ dimensions, that the index of the Dirac operator does not change under small deformations of it, and that in our formalism we are considering small deformations of the ordinary Dirac operator. Putting it all together: no $\theta$-dependent anomalous terms.

- A challeging question: Will these noncommutative GUTs eventually find accommodation within F-theory [26]?

- A final question: can one formulate noncommutative GUTs without using the envelopingalgebra formalism? In answering this question in the affirmative, the ideas presented in refs. [27, 28] look most promising; see also [29] .

\section{Acknowledgements}

I should like to thank the organizers -D. Bahns, H. Grosse and G. Zoupanos- of this Workshop for giving me the opportunity to present the material covered in this talk at this wonderful conference held in a place with such beautiful surroundings. This work has been financially supported in part by MICINN through Grant No. FPA2008-04906.

\section{References}

[1] J. Madore, S. Schraml, P. Schupp and J. Wess, Gauge theory on noncommutative spaces, Eur. Phys. J. C 16 (2000) 161 [arXiv: hep-th/ 0001203 ].

[2] B. Jurco, P. Schupp and J. Wess, Noncommutative gauge theory for Poisson manifolds, Nucl. Phys. B 584 (2000) 784 [arXiv: hep-th/0005005].

[3] B. Jurco, S. Schraml, P. Schupp and J. Wess, Enveloping algebra valued gauge transformations for non-Abelian gauge groups on non-commutative spaces, Eur. Phys. J. C 17 (2000) 521 [arXiv:hep-th/0006246].

[4] X. Calmet, B. Jurco, P. Schupp, J. Wess and M. Wohlgenannt, The standard model on non-commutative space-time, Eur. Phys. J. C 23 (2002) 363 [arXiv: hep-ph/0111115].

[5] P. Aschieri, B. Jurco, P. Schupp and J. Wess, Non-commutative GUTs, standard model and C, P, T, Nucl. Phys. B 651 (2003) 45 [arXiv: hep-th/ 0205214 ]. 
[6] D. N. Blaschke, E. Kronberger, R. I. P. Sedmik and M. Wohlgenannt, Gauge Theories on Deformed Spaces, SIGMA 6 (2010) 062 [arXiv:1004.2127 [hep-th] ].

[7] P. Schupp, Non-Abelian gauge theory on noncommutative spaces, [arXiv: hep-th/0111038].

[8] C. P. Martin, The gauge anomaly and the Seiberg-Witten map, Nucl. Phys. B 652 (2003) 72 [arXiv:hep-th/0211164].

[9] G. Barnich, F. Brandt and M. Henneaux, Local BRST cohomology in gauge theories, Phys. Rept. 338 (2000) 439 [arXiv: hep-th/0002245].

[10] F. Brandt, C. P. Martin and F. R. Ruiz, Anomaly freedom in Seiberg-Witten noncommutative gauge theories, JHEP 0307 (2003) 068 [arXiv: hep-th/ 0307292 ].

[11] A. Bichl, J. Grimstrup, H. Grosse, L. Popp, M. Schweda and R. Wulkenhaar, Renormalization of the noncommutative photon self-energy to all orders via Seiberg-Witten map, JHEP 0106 (2001) 013 [arXiv:hep-th/0104097].

[12] R. Wulkenhaar, Non-renormalizability of Theta-expanded noncommutative QED, JHEP $\mathbf{0 2 0 3}$ (2002) 024 [arXiv:hep-th/0112248].

[13] M. Buric, D. Latas and V. Radovanovic, Renormalizability of noncommutative $S U(N)$ gauge theory, JHEP 0602 (2006) 046 [arXiv : hep-th / 0510133 ].

[14] M. Buric, V. Radovanovic and J. Trampetic, The one-loop renormalization of the gauge sector in the noncommutative standard model, JHEP 0703 (2007) 030 [arXiv: hep-th / 0609073 ].

[15] M. Buric, D. Latas, V. Radovanovic and J. Trampetic, The absence of the $4 \psi$ divergence in noncommutative chiral models, Phys. Rev. D 77 (2008) 045031 [arXiv: 0711.0887 [hep-th] ].

[16] C. P. Martin and C. Tamarit, Noncommutative GUT inspired theories and the UV finiteness of the fermionic four point functions, Phys. Rev. D 80 (2009) 065023 [arXiv: 0907.2464 [hep-th]].

[17] C. P. Martin and C. Tamarit, Renormalisability of noncommutative GUT inspired field theories with anomaly safe groups, JHEP 0912 (2009) 042 [arXiv:0910.2677 [hep-th] ].

[18] C. Tamarit, Noncommutative GUT inspired theories with $U(1), S U(N)$ groups and their renormalisability, Phys. Rev. D 81 (2010) 025006 [arXiv: 0910.5195 [hep-th] ].

[19] C. P. Martin, Yukawa terms in noncommutative SO(10) and E6 GUTs, Phys. Rev. D 82 (2010) 085020 [arXiv:1008.1871 [hep-ph]].

[20] C. P. Martin and C. Tamarit, The Seiberg-Witten map and supersymmetry, JHEP 0811 (2008) 087 [arXiv:0809.2684 [hep-th]].

[21] C. P. Martin and C. Tamarit, Noncommutative N=1 super Yang-Mills, the Seiberg-Witten map and UV divergences, JHEP 0911 (2009) 092 [arXiv:0907.2437 [hep-th] ].

[22] S. Paban, S. Sethi and M. Stern, Non-commutativity and supersymmetry, JHEP 0203 (2002) 012 [arXiv:hep-th/0201259].

[23] V. Putz and R. Wulkenhaar, Seiberg-Witten map for noncommutative super Yang-Mills theory, Int. J. Mod. Phys. A 18 (2003) 3325 [arXiv: hep-th/0205094].

[24] O. F. Dayi, K. Ulker and B. Yapiskan, Duals of noncommutative supersymmetric U(1) gauge theory, JHEP 0310 (2003) 010 [arXiv: hep-th/ 0309073$].$

[25] D. Mikulovic, Seiberg-Witten map for superfields on canonically deformed $N=1, d=4$ superspace, JHEP 0401 (2004) 063 [arXiv : hep-th/ 0310065 ]. 
[26] S. Cecotti, M. C. N. Cheng, J. J. Heckman and C. Vafa, Yukawa Couplings in F-theory and Non-Commutative Geometry, [arXiv:0910.0477 [hep-th]].

[27] H. Grosse, F. Lizzi and H. Steinacker, Noncommutative gauge theory and symmetry breaking in matrix models, Phys. Rev. D 81 (2010) 085034 [arXiv: 1001.2703 [hep-th] ].

[28] A. Chatzistavrakidis, H. Steinacker and G. Zoupanos, Orbifolds, fuzzy spheres and chiral fermions, JHEP 1005 (2010) 100 [arXiv: 1002.2606 [hep-th] ].

[29] L. Bonora, M. Schnabl, M. M. Sheikh-Jabbari and A. Tomasiello, Noncommutative $S O(n)$ and $S p(n)$ gauge theories, Nucl. Phys. B 589 (2000) 461 [arXiv: hep-th/ 0006091 ]. 